GA-A23758

\title{
RECENT PROGRESS IN FABRICATION OF HIGH- STRENGTH GLOW DISCHARGE POLYMER SHELLS BY OPTIMIZATION OF COATING PARAMETERS
}

by

A. NIKROO, D.G. CZECHOWICZ, E.R. CASTILLO, and J.M. PONTELANDOLFO 


\section{DISCLAIMER}

This report was prepared as an account of work sponsored by an agency of the United States Government. Neither the United States Government nor any agency thereof, nor any of their employees, makes any warranty, express or implied, or assumes any legal liability or responsibility for the accuracy, completeness, or usefulness of any information, apparatus, product, or process disclosed, or represents that its use would not infringe privately owned rights. Reference herein to any specific commercial product, process, or service by trade name, trademark, manufacturer, or otherwise, does not necessarily constitute or imply its endorsement, recommendation, or favoring by the United States Government or any agency thereof. The views and opinions of authors expressed herein do not necessarily state or reflect those of the United States Government or any agency thereof. 


\title{
RECENT PROGRESS IN FABRICATION OF HIGH- STRENGTH GLOW DISCHARGE POLYMER SHELLS BY OPTIMIZATION OF COATING PARAMETERS
}

\author{
by \\ A. NIKROO, D.G. CZECHOWICZ, E.R. CASTILLO, and \\ J.M. PONTELANDOLFO
}

This is a preprint of a paper to be presented at the 14th Target Fabrication Meeting, July 15-19, 2001, West Point, New York and to be published in Fusion Technology.

\author{
Work supported by \\ the U.S. Department of Energy \\ under Contract No. DE-AC03-01SF22260
}




\title{
RECENT PROGRESS IN FABRICATION OF HIGH-STRENGTH GLOW DISCHARGE POLYMER SHELLS BY OPTIMIZATION OF COATING PARAMETERS
}

\author{
A. Nikroo, D.G. Czechowicz, E.R. Castillo, J.M. Pontelandolfo \\ Inertial Fusion Division \\ General Atomics, P.O. Box 85608, San Diego, California 92186-5608
}

(858) 455-2931, email: abbas.nikroo@gat.com

\begin{abstract}
In this paper, we report the progress we have made in fabrication of high-strength thin-walled glow discharge polymer (GDP) shells for cryogenic experiments at OMEGA. We have investigated a number of different parameters involved in making such shells. Optimization of hydrogen to hydrocarbon precursor flow has been observed to be critical in obtaining strong shells. We can routinely make high-strength shells of OMEGA size $(900 \mu \mathrm{m}$ in diameter) with thicknesses in the range of 1.0 to $1.5 \mu \mathrm{m}$. The permeabilities of these shells to various gases have been found to be as much as three times higher than those of lower strength shells. Run to run variability and other batch statistics will be discussed.
\end{abstract}

\section{INTRODUCTION}

Thin walled polymer shells are needed for OMEGA cryogenic laser experiments. The capsules need to be about $900 \mu \mathrm{m}$ in diameter and as thin as possible $(\approx 1 \mu \mathrm{m})$ while having sufficient strength to be filled with $\mathrm{D}-\mathrm{T}$ as fast as possible to about $1000 \mathrm{~atm}$. This requires that these high aspect ratio shells have high buckle strength and permeability. Shell strength is also important because they need to survive routine handling required for characterization and assembly. We have previously reported on fabrication of such high aspect ratio shells using the PAMS/GDP process and the possibility of strengthening the shells by changing the GDP coating parameters. ${ }^{1}$ In that study, we had found that shell strength depended strongly on deposition pressure. Using lower deposition pressures we were able to increase the strength of thin wall shells by as much as a factor of two. In this paper, we report on the effects of various other deposition parameters on shell strength. In particular, we have found that gas flow ratios, at the lower deposition pressures, can have a dramatic effect on the strength of shells. Optimization of gas flow ratios at low deposition pressures allows routine fabrication of stronger thin wall GDP shells. In this paper, we also discuss the effects of several other deposition parameters, permeability measurements, and reproducibility of the fabrication process.

\section{EXPERIMENTAL}

Thin walled GDP shells were made by the depolymerizable mandrel technique using poly-alpha-methylstyrene (PAMS) mandrels. ${ }^{2}$ The GDP deposition system hardware has been described previously in the literature. ${ }^{3}$ This system is used on a routine basis to fabricate capsules for ICF experiments. ${ }^{4}$ In this process, trans-2-butene is mixed with an excess of hydrogen, the mixture is broken down in the plasma region and the hydrocarbon fragments deposit on the substrates forming ultra-smooth coatings. For this study, in order to deposit at low pressures and high hydrogen flows, a new pumping system was placed very close to the coater to provide the required pumping speed. This greatly expanded the useable parameter space of the system. In a number of cases, multiple runs using the same coating conditions were performed to verify the reproducibility of the coatings.

Buckle pressures were measured using the device described previously. ${ }^{1}$ Briefly, a pre-chamber is pressurized to the desired test pressure. Shells are then subjected to this pressure in a fraction of a second to avoid filling the shells as they are tested. A camera allows observation of shell buckling directly. This device was upgraded for this study, including the addition of a fine control regulator capable of reaching $50 \mathrm{psi}$ in $0.1 \mathrm{psi}$ steps. We estimate an error of $<3 \%$ for buckle pressure 
measurements. Shell diameters were measured using the standard characterization techniques used in our laboratory. The diameter could be measured to $\leqslant 1 \mu \mathrm{m}$, resulting in an error of $<1 \%$ when measuring $900 \mu \mathrm{m}$ shells. Using interferometry, we normally measure shell wall thickness to about $0.1 \mu \mathrm{m}$. For a $1 \mu \mathrm{m}$ thick shell, this translates into a $10 \%$ error which propagates into a $20 \%$ error in the Young's Modulus. This represents the largest error in determining the Young's Modulus. To verify the wall thickness values found by interferometry, we also used destructive scanning electron microscope (SEM) measurements for many of the batches. The comparison is shown in Fig. 1. The agreement was to about $0.1 \mu \mathrm{m}(<10 \%)$ for almost all batches.

\section{RESULTS AND DISCUSSION}

The theoretical buckle strength of an ideal defect-free spherical shell of wall thickness $w$ and diameter $d$, can be found using the expression, 1

$$
E=\frac{\sqrt{3\left(v^{2}-1\right)}}{8} P_{b}\left(\frac{D}{w}\right)^{2},
$$

Where $E$ and $v$ are the Young's Modulus and Poisson's ratio for the material, respectively. Therefore, to perform a comparison of buckle strength of shells made under different conditions, any difference in the aspect ratio, $D / w$, needs to be properly accounted for. We have done this by calculating the Young's Modulus and using this value when comparing the strength of different batches of shells with different aspect ratios. $v$ was assumed to be 0.30 , typical for polymers. Others and we have tested the validity of this procedure for wall thicknesses greater than $\approx 1 \mu \mathrm{m} .{ }^{1,6}$ Since, in practice, most shells are not defect free, this procedure serves to remove

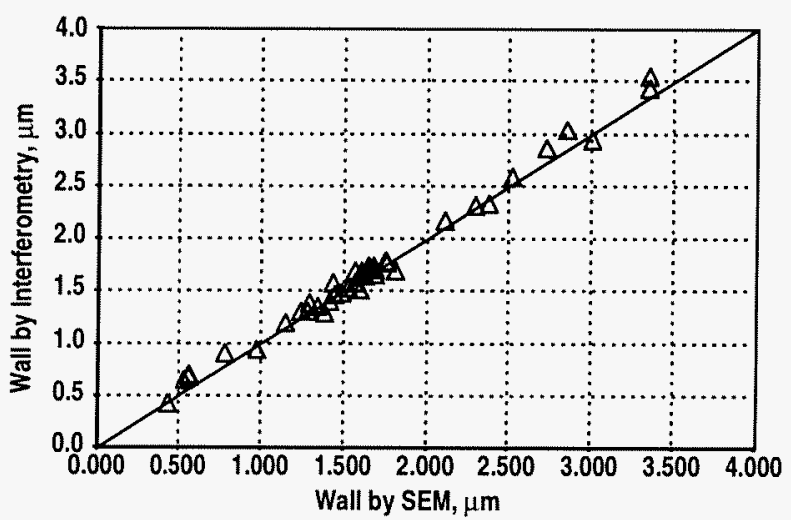

Fig. 1. Comparison of wall thickness measurements by interferometry and SEM. SEM measurements were used to verify values obtained by interferometry. any aspect ratio dependence from the measurements, rather than determining a true Young's Modulus for the deposited films.

\section{A. Effect of Coating Gases}

We studied the effect of gas flow ratios in the GDP process. We performed a number of runs varying the hydrogen $\left(\mathrm{H}_{2}\right)$ to trans-2-butene (T2B) flow ratio. The coatings were deposited at 20,25, 40 and 75 mTorr. At first, $\mathrm{H}_{2}$ flow was kept constant at $2.0 \mathrm{sccm}$ while T2B flow was varied between 0.08 and $0.32 \mathrm{sccm}$. The $\mathrm{H}_{2} / \mathrm{T} 2 \mathrm{~B}$ ratio had a dramatic effect on the strength of the shells at the lowest deposition pressures as shown in Fig. 2. The higher $\mathrm{H}_{2} / \mathrm{T} 2 \mathrm{~B}$ flow ratio resulted in higher strength shells. The effect was more pronounced at 20 and 25 mTorr. Using optimized flow ratio we could routinely make $\approx 1 \mu \mathrm{m}$ thick shells with Young's Moduli of over $2.3 \mathrm{GPa}$. In practical terms, this translates into a buckle pressure of $2.0 \mathrm{psi}$ for a $1 \mu \mathrm{m}$ thick, $900 \mu \mathrm{m}$ diameter shell. This is comparable to values reported for thin walled polyimide shells of similar dimensions made using vapor deposition. ${ }^{7}$ At the highest $\mathrm{H}_{2} / \mathrm{T} 2 \mathrm{~B}$ ratios and at 20 and 25 mTorr coating pressures, the PAMS shells disintegrated rapidly (Fig. 2). This is probably due to lack of sufficient hydrocarbon precursor in the coater to form a coating and the resulting etching of the PAMS shells by hydrogen (or residual oxygen) in the coating chamber. This, therefore, placed an upper limit on attainable shell strength.

We also explored higher $\mathrm{H}_{2}$ flows at constant, rather high $(0.16 \mathrm{sccm})$, T2B flow and observed the same increase in shell strength with higher $\mathrm{H}_{2}$ /T2B flows. In this case, at the much higher $\mathrm{H}_{2} / \mathrm{T} 2 \mathrm{~B}$ flows, the PAMS

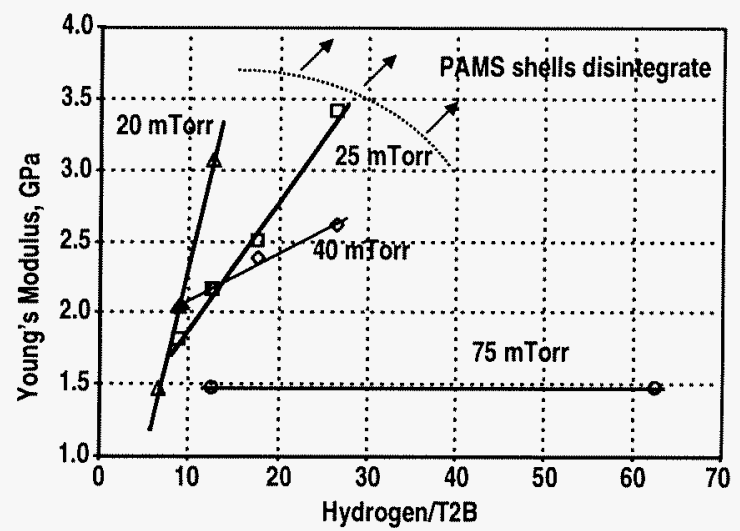

Fig. 2. The hydrogen-to-T2B flow ratio used in the GDP coating had a dramatic effect on the strength of the final GDP shells. This was true only for the lowest deposition pressures where the strongest shells are usually made. PAMS shells disintegrated for flow ratios above a certain point possibly due to hydrogen etching and lack of hydrocarbon species. 
shells did not disintegrate as they had at the lower T2B flows. Apparently, the T2B flow was high enough to obtain a coating despite the high $\mathrm{H}_{2}$ flow. However, the strength of the resulting shells began to decrease for $\mathrm{H}_{2} / \mathrm{T} 2 \mathrm{~B}$ ratios higher than those shown in Fig. 2, indicating that maximum strength is obtained at an optimum flow ratio. The runs involving the higher $\mathrm{H}_{2}$ flow had the added benefit that the coating on the inside of the plasma tube appeared to be more stable and did not flake onto the shells during the run. This flaking is a common problem for runs at low pressures and low $\mathrm{H}_{2}$ and T2B flows. The absence of such flaking in the higher $\mathrm{H}_{2}$ runs allowed longer runs to make thicker shells $(>2 \mu \mathrm{m})$ if needed without multiple tube changes during the run. This proved to be very useful for production of $3 \mu \mathrm{m}$ thick shells needed for experiments on OMEGA.

Surface finish of shells also improved as the T2B flow was decreased at constant $\mathrm{H}_{2}$ flow. Figures 3(a) and (b) show atomic force microscope (AFM) patch and spheremap ${ }^{8}$ scans of shells made using varying $\mathrm{H}_{2} / \mathrm{T} 2 \mathrm{~B}$ ratios. The surface finish improvement with increasing $\mathrm{H}_{2} / \mathrm{T} 2 \mathrm{~B}$ flow ratio is well known in our GDP deposition system and had been previously attributed to the lowering of T2B residence time. ${ }^{9}$ However, in this case, the constant $\mathrm{H}_{2}$ flow results in constant T2B residence time due to the much higher $\mathrm{H}_{2}$ flow. Therefore, the etching effect of $\mathrm{H}_{2}$, hypothesized in diamond-like coating literature, 10 is the most likely cause of the simultaneous surface finish improvement and higher strength of the films.

We investigated replacing $\mathrm{T} 2 \mathrm{~B}$, the usual hydrocarbon precursor used for GDP coatings, with first methane and then benzene. As with T2B, we were able to make shells with Young's Moduli over $2.3 \mathrm{GPa}$ with these precursors. The coating parameters had to be optimized in each case. The strongest shells made using methane were $10 \%$ to $20 \%$ weaker than the strongest shells made using T2B. Relative hydrogen flow had a large effect on the strength of shells made using methane similar to the effect observed for T2B. For benzene, while there appeared to be a similar trend, the results were not as reproducible. Several runs, using benzene as the precursor, produced shells with Young's Moduli over $4.0 \mathrm{GPa}$, the highest value obtained to date. However, other runs using identical parameters resulted in shells with much lower moduli. Coatings involving benzene have been much less studied than the other two precursors. We hope to investigate benzene as a precursor more thoroughly in the future.

\section{B. Permeability Measurements}

A major goal of seeking stronger shells is to allow thin-walled shells to be permeation filled more quickly. A major concern, therefore, was whether the stronger GDP shells might have lower permeability. Permeability measurements for $\mathrm{He}, \mathrm{Ne}$ and $\mathrm{N}_{2}$ were made destructively using buckle strengths measured for the batch. Ar and Xe permeabilities were measured using $\mathrm{X}$-ray fluorescence (XRF). A homemade permeation cell was also used for permeability measurements for the various gases. The details of these measurements will be the subject of a future publication. Numerous permeability measurements of the shells made using T2B against various gases such $\mathrm{He}, \mathrm{Ne}, \mathrm{N}_{2}$ and Xe indicate that the stronger shells are indeed more permeable than the normal GDP shells (Fig. 4). The stronger GDP shells were found to be several times more permeable than normal GDP shells. A number of these shells from various batches were also measured independently at UR/LLE for both buckle strength and permeability. Those measurements confirmed the higher permeability of the higher modulus shells to $\mathrm{N}_{2}$ and $\mathrm{Ar}$
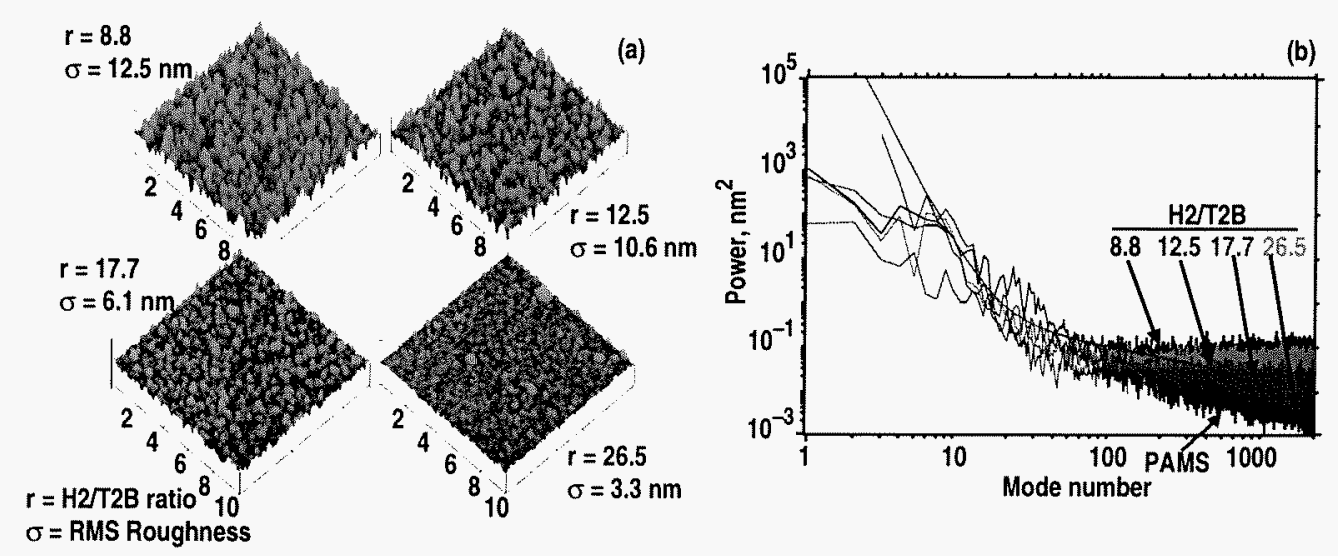

Fig. 3. (a) Atomic force microscope patch scans of surfaces of $\approx 2 \mu \mathrm{m}$ GDP shells. The higher hydrogen-to-T2B ratio results in smoother surfaces. These coatings were deposited at $40 \mathrm{mTorr}$. (b) AFM spheremaps of the shells shown in (a). The low mode power ( $<100)$ is mainly due to the starting PAMS shell. The higher mode power ( $>100)$ is dominated by the hydrogen to T2B flow ratio (shown in Fig. 3(a) also). The bare PAMS shell spectrum is also shown for comparison. 


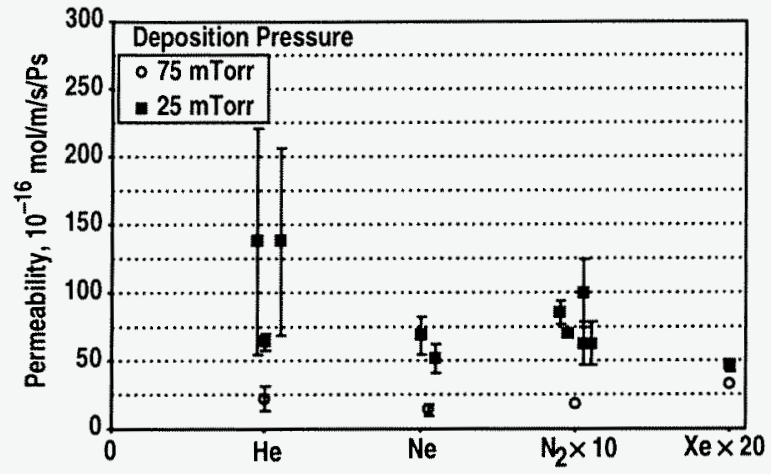

Fig. 4. The stronger shells made at the lower deposition pressures were more permeable to a number of different gases. Standard GDP shells are made at deposition pressure of $75 \mathrm{~m}$ Torr. The higher permeability is highly desirable and allows filling shells faster.

and confirmed our buckle strength data. $\mathrm{D}_{2}$ permeability still needs to be measured for these shells. However, due to the very short half-life of this gas for thin wall shells, the measurements will be difficult.

\section{Other Parameters}

We also examined the effects of using different PAMS batches on the strength of the final GDP shells. A PAMS batch used in our previous study was used as a control batch in order to account for possible run-to-run variations in the GDP process. Shells from this batch were coated and pyrolyzed together with shells from the batch, which was being tested. This was done with seven different PAMS batches. The results are plotted in Fig. 5. The most interesting conclusion is that some PAMS batches result in GDP shells with very tight ranges (5\% standard deviation) of buckle pressures, while others had much broader ranges ( $20 \%$ standard deviation). In addition, one batch resulted in shells, which were as much as $20 \%$ stronger than the control batch. The tight range of buckle pressures allows testing a small sample of shells from a batch to determine the typical buckle strength for the batch with a high degree of confidence. This leaves the rest of the shells from the batch for other measurements such as permeability. Reproducibility and the spread of strength values observed within a given batch are discussed below.

We briefly examined some of the other parameters that might affect coating strength. We varied the pyrolysis time. Doubling the pyrolysis time did not affect the strength of shells. We also examined using magnets to confine the coating plasma. The magnet configuration used appeared to result in localized confinement only, which did not benefit shells bouncing over larger areas in

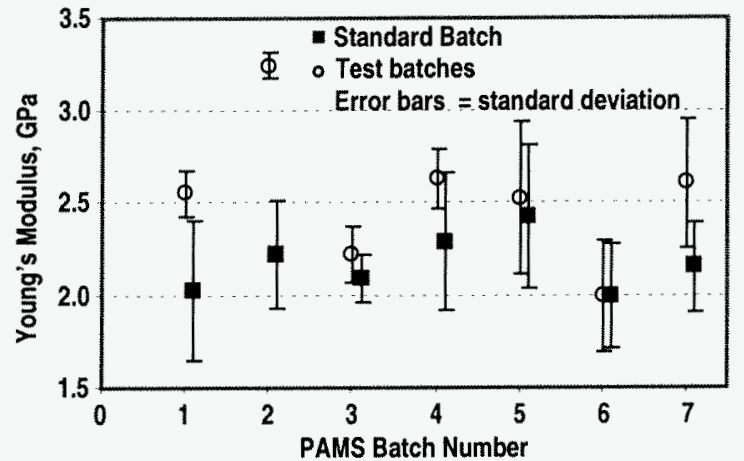

Fig. 5. The effect of the starting PAMS batch on the strength of the final GDP shells was examined. A standard batch was coated and pyrolyzed along with the standard batch, which was being tested to account for possible run-to-run variations in GDP coating runs.

the coating pan. Consequently, shells made using this technique did not have as high a modulus as without confinement. Radio Frequency (rf) biasing of substrates was also examined. Since our main coating plasma is powered by only $10 \mathrm{~W}$, the biasing field (tens of watts) could create its own secondary plasma. This led to instability in the operation of the coater and the desired bias level. Low bias $(\leq 10 \mathrm{~V})$ levels could be stably sustained, but this did not affect strength of shells. Shells made at higher bias values typically collapsed in the pyrolysis process. A different, slightly modified helical resonator (plasma generator) was also used for making high modulus shells. By optimizing the coating parameters, we were again able to make shells with moduli over $2.3 \mathrm{GPa}$ using the second helical resonator. This illustrated the lack of dependence of the process on the particular coating system used.

\section{Composition, Aging and Reproducibility}

We determined the chemical composition of our films using combustion analysis. The carbon-to-hydrogen ratio in the high modulus films is about 1.16 while for normal GDP (lower modulus) it is only 0.85 . The lower hydrogen content may explain the higher moduli of films made under modified conditions. The oxygen content of the higher modulus films was also higher (5.5 atomic \%) compared to normal GDP ( 3.0 atomic \%). The density of fresh (minimal exposure to oxygen) higher modulus films was measured to be $1.20 \mathrm{~g} / \mathrm{cc}$ compared to $1.04 \mathrm{~g} / \mathrm{cc}$ for normal GDP. This is again consistent with the higher carbon content of higher modulus films. This higher density can be of value for indirect drive NIF capsules as well. Film composition was also examined by infrared spectroscopy and the results are presented in a separate publication. ${ }^{11}$ 
We examined the effects of air or nitrogen storage on the buckle strength of thin GDP shells. Oxygen uptake of GDP films upon air exposure is a well-known effect. 11,12 This is an important issue, since shells fabricated for cryogenic experiments at UR/LLE will eventually have to be stored for a number of weeks before they are used. A number of different GDP shell batches containing at least 30 shells were made. Each batch was divided equally into four groups. One group was tested immediately. The other three groups were stored under nitrogen for some of the batches and under air for some of the other batches. These groups were subsequently buckle tested after one, two and four weeks of air or nitrogen storage, respectively. The results are shown in Fig. 6. The data indicates that within the variations seen in each group air or nitrogen storage did not have a significant effect on the buckle strength of GDP shells in this period of time. However, more recent data indicates that longer air exposure may indeed lead to weakening of shells and reduced permeability. Therefore, this issue needs to be studied further.

Figure 7 shows the results of 10 different runs made using exactly the same optimized GDP run conditions, PAMS batch, and pyrolysis procedure. The error bars indicate the entire range of buckle strengths measured for a minimum of 8 shells from each batch. As can be seen from the figure for most of the batches the range of observed buckle strengths is very small $(<10 \%)$. Also the batches with Young's Modulus values of over 3.0 GPa were also made at times. GDP run-to-run variation is the most likely cause of variations in the observed Young's Modulus values.

\section{CONCLUSION}

We have made major advances in developing strong thin-walled GDP capsules. By optimizing the coating parameters, we can achieve over a factor of 2 increase in the normalized buckling pressure (over 2.0 psi for a $1 \mu \mathrm{m}$

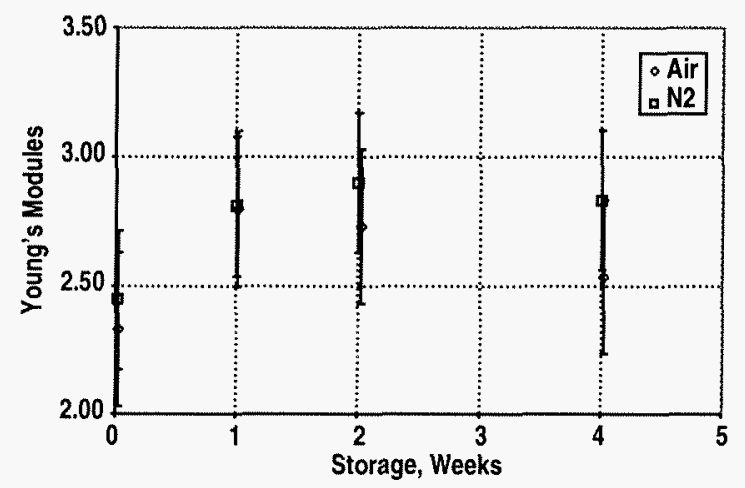

Fig. 6. Air and nitrogen storage of thin-walled GDP shells was examined. Buckle strength of shells did not change on storage in air or nitrogen over a four-week period.

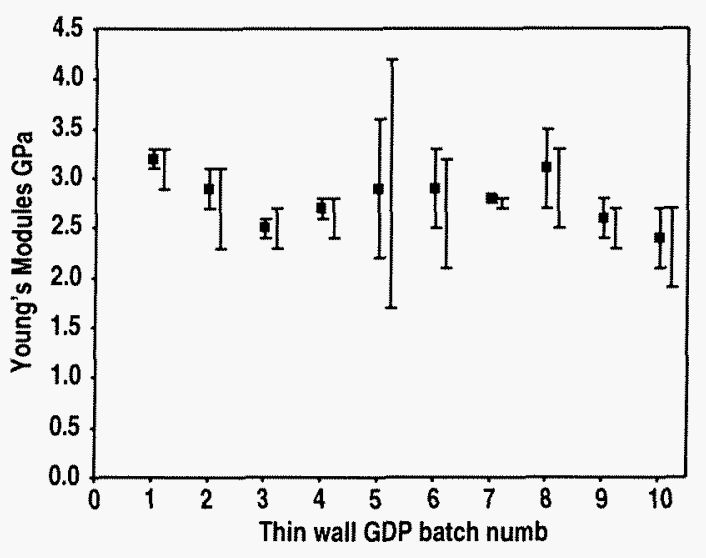

Fig. 7. Run-to-run variability and spread of Young's Modulus values within a batch are shown for 10 different batches of shells made using identical processing conditions. The solid squares indicate the average for at least 10 shells from the batch, with the error bars around them indicating the standard deviation for the measured shells. The bare error bars indicate the entire range of buckle strengths measured for the 10 shells from each batch.

thick, $900 \mathrm{~mm}$ diameter shell) compared to the usual GDP capsules. This high strength material is several times more permeable than normal GDP to a number of different gases. Shells made from this material can therefore be permeation filled several times faster. The strength of this high-strength GDP is comparable to that of vapor deposited polyimide. The good surface finish that can be achieved with GDP capsules is retained with the high strength material. Further optimization and improvement may be possible and should be pursued.

\section{ACKNOWLEDGMENT}

Work supported by the U.S. Department of Energy under Contract DE-AC03-01SF22260.

\section{REFERENCES}

1. A. Nikroo, J. Pontelandolfo, "Fabrication of Thin Walled Glow Discharge Polymer Shells," Fusion Technol., 38, 58 (2000).

2. B.W. McQuillan, A. Nikroo, D.A. Steinman, F.H. Elsner, D.G. Czechowicz, M.L. Hoppe, M. Sixtus, and W.J. Miller, "The PAMS/GDP Process for Production of ICF Target Mandrels" Fusion Technol., 31, 381 (1997).

3. S.W. Ferguson, R. Stever, and R.M. Brusasco, "Impedence-Power Effects on Plasma Polymer Surface Finish Using a Helical Resonator Discharge," J. Appl. Polym. Sci., 54, 107 (1994).

4. A. Nikroo et al., "Capsule Development and Production for Inertial Confinement Fusion Experiments," Proc. of the First Conference on 
Inertial Fusion Sciences and Applications 1999, edited by C. Labaune, W.J. Hagan, K.A. Tanaka (Elsevier Press, Paris, 2000) 917.

5. R.J. Roark, W.C. Young, "Formulas for Stress and Strain," McGraw-Hill Book Company, 5th Edition, 557 (1989).

6. Laboratory for Laser Energetics Review 81, 6 (2000).

7. F.Y. Tsai, E.L. Alfonso, S.H. Chen, D.R. Harding, "Mechanical Properties and Gas Permeability of Polyimide Shells Fabricated by the Vapor Deposition Method," Fusion Technol., 38, 83 (2000).

8. R.L. McEachren, C.E. Moore, R.J. Wallace, "The Design, Performance, and Application of an
Atomic Force Microscope-Based Profilometer," J. Vac. Sci. Technol. A 13, 983 (1995).

9. S.A. Letts, D.W. Meyers, and L.A. Witt, J. Vac. Sci. Technol. 19, 739 (1981).

10. Y. Cathrine, "Deposition of Amorphous Yydrogenated Carbon Films in Low and High Frequency Discharges," Mater. Sci. Forum Vols. 52, 53, 175 (1989).

11. D.G. Czechowicz, E.R. Castillo, A. Nikroo, "Compositional and Structural Studies of Glow Discharge Polymer Coatings," these proceedings.

12. T.R. Gengenbach, Z.R. Vasic, R.C. Chateiler, and H.J. Griesser, "A Multi-Technique Study of the Spontaneous Oxidation of n-Hexane Plasma Polymer," J. Polym. Sci. A 32, 1399 (1994). 